\title{
Study of the primordial Lithium Abundances at CIAE
}

Z. H. Li*, E. T. Li, J. Su, B. Guo, W. P. Liu, Y. J. Li, Y. S. Chen, S. Q. Hou, X. X. Bai, S. Q. Yan, Y. B. Wang, B. X. Wang, S. Zeng, G. Lian, X. Liu, S. J. Jin

China Institute of Atomic Energy, Beijing 102413, China

E-mail: Zh]iबciae.ac.c口

Lithium isotopes have attracted an intense interest because the predicted abundances of both ${ }^{6} \mathrm{Li}$ and ${ }^{7} \mathrm{Li}$ from big bang nucleosynthesis (BBN) do not match experimental observations. Many investigations of astrophysical observations and BBN calculations have been carried out in order to solve the puzzles. Several nuclear reactions involving lithium have been determined at HI-13 tandem accelerator, Beijing, China. The BBN model calculations are then performed to investigate the primordial lithium abundance. The result shows that these nuclear reactions have minimal effect on the primordial abundances of ${ }^{6} \mathrm{Li}$ and ${ }^{7} \mathrm{Li}$.

XII International Symposium on Nuclei in the Cosmos,

August 5-12, 2012

Cairns, Australia

\footnotetext{
* Speaker.

$\dagger$ This work is supported by the National Natural Science Foundation of China under Grant Nos. 11021504 and 10975193, the National Basic Research Programme of China under Grant No. 2013CB834406.
} 


\section{Introduction}

Since the pioneering work of M. Spite and F. Spite [四], the lithium abundance in the metalpoor halo stars was confirmed as a plateau, independent of metallicity and effective temperature. Up to now, the most widely accepted interpretation is that the lithium observed in metal-poor stars has been produced in the big bang nucleosynthesis (BBN). According to the standard Big Bang model, the relative abundances of the light elements $\left({ }^{1} \mathrm{H},{ }^{2} \mathrm{H},{ }^{3} \mathrm{He},{ }^{4} \mathrm{He},{ }^{6} \mathrm{Li}\right.$, and $\left.{ }^{7} \mathrm{Li}\right)$ depend on only one parameter, namely, the baryon to photon ratio $\eta$. Using the precisely determined $\eta$ from cosmic microwave background fluctuations, the lithium to hydrogen ratio is predicted to be ${ }^{7} \mathrm{Li} / \mathrm{H}$ $=(4.2 \pm 0.5) \times 10^{10}[[]$, which is higher than that observed in metal poor halo stars by roughly a factor of three. Even worse, the recent claims of detection of isotope-shifted lithium absorption lines in a subset of the stars point to a ${ }^{6} \mathrm{Li}$ abundance some three orders of magnitude larger than that expected in BBN model [B]].

In order to solve the lithium problems from nuclear physics aspect, several nuclear reactions involving lithium, such as ${ }^{6} \mathrm{Li}(n, \gamma){ }^{7} \mathrm{Li},{ }^{7} \mathrm{Li}(n, \gamma){ }^{8} \mathrm{Li},{ }^{8} \mathrm{Li}(n, \gamma){ }^{9} \mathrm{Li},{ }^{6} \mathrm{Li}(p, \gamma){ }^{7} \mathrm{Be},{ }^{6} \mathrm{He}(p, \gamma){ }^{7} \mathrm{Li}$, ${ }^{6} \mathrm{He}(p, n){ }^{6} \mathrm{Li},{ }^{6} \mathrm{He}(d, n){ }^{7} \mathrm{Li},{ }^{8} \mathrm{Li}(p, \gamma){ }^{9} \mathrm{Be},{ }^{8} \mathrm{Li}(p, d){ }^{7} \mathrm{Li},{ }^{8} \mathrm{Li}(p, t){ }^{6} \mathrm{Li},{ }^{8} \mathrm{Li}(d, p){ }^{9} \mathrm{Li},{ }^{8} \mathrm{Li}(d, n){ }^{9} \mathrm{Be},{ }^{7} \mathrm{Be}(d$, $\left.{ }^{3} \mathrm{He}\right){ }^{6} \mathrm{Li}$, and ${ }^{9} \mathrm{Be}(p, \alpha){ }^{6} \mathrm{Li}$ were measured at $\mathrm{HI}-13$ tandem accelerator, Beijing. The rates of these nuclear reactions were deduced and then used in the BBN network calculations.

\section{Experiments}

Figure $\mathbb{W}$ shows the reactions network of BBN, which is modified from the standard BBN model code [ [G]. The reactions labeled with the dashed line are determined at China institute of Atomic energy in about ten years.

The $(n, \gamma)$ reactions were determined by measuring the angular distributions of single neutron transfer reactions. Where, the ${ }^{6} \mathrm{Li}(n, \gamma)^{7} \mathrm{Li}$ excitation function was deduced from the elastic transfer reaction of ${ }^{7} \mathrm{Li}\left({ }^{6} \mathrm{Li},{ }^{7} \mathrm{Li}\right){ }^{6} \mathrm{Li}\left[\right.$ [ []]. The ${ }^{6} \mathrm{Li}(n, \gamma){ }^{7} \mathrm{Li}$ rates were derived to be $(8.1 \pm 0.6) \times 10^{3}$ $\mathrm{cm}^{3} \mathrm{~mol}^{-1} \mathrm{~s}^{-1}$ at the energies of astrophysical interests. The angular distribution of ${ }^{13} \mathrm{C}\left({ }^{7} \mathrm{Li},{ }^{8} \mathrm{Li}\right){ }^{12} \mathrm{C}$

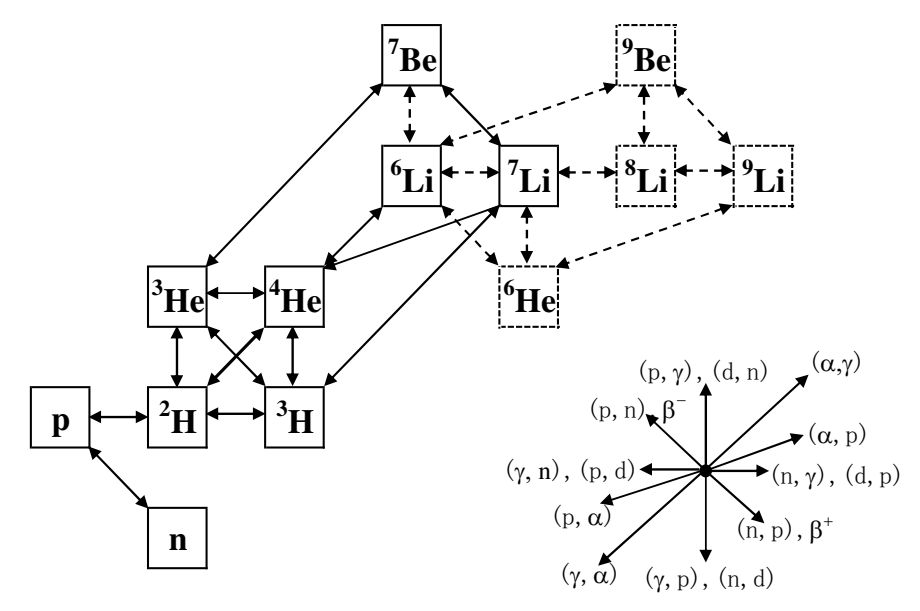

Figure 1: Reaction network for BBN calculation in the present work, as modified from Ref. [四]. 
was measured and used to extract the ${ }^{8} \mathrm{Li}$ neutron spectroscopic factor with Distorted-Wave Born Approximation (DWBA) analysis. The ${ }^{7} \mathrm{Li}(n, \gamma)^{8} \mathrm{Li}$ cross sections are then deduced using the direct capture model calculations [可]. The angular distribution of the ${ }^{8} \mathrm{Li}(d, p)^{9} \mathrm{Li}$ reaction was measured at $E_{\text {c.m. }}=7.8 \mathrm{MeV}$ in inverse kinematics using coincidence detection of ${ }^{9} \mathrm{Li}$ and the recoil proton. The neutron spectroscopic factor $S_{1,3 / 2}$ for ${ }^{9} \mathrm{Li} \rightarrow{ }^{8} \mathrm{Li}+\mathrm{n}$ was derived to be $0.68 \pm 0.14$. The astrophysical ${ }^{8} \mathrm{Li}(n, \gamma){ }^{9} \mathrm{Li}$ reaction rate for the direct capture was then deduced to be $3970 \pm 950$ $\mathrm{cm}^{3} \mathrm{~mole}^{-1} \mathrm{~s}^{-1}$ at $T_{9}=1[$ [ $]$.

The $(p, \gamma)$ reactions play important roles in nuclear astrophysics, but it is very difficult to measure directly due to the small cross section at the energy of astrophysical interests. We determined the excitation function of $(p, \gamma)$ from the measured angular distribution of one proton transfer reaction. The proton spectroscopic factor of ${ }^{7} \mathrm{Li}$ was extracted to be $0.42 \pm 0.06$ by normalizing the calculated differential cross sections with the distorted-wave Born approximation to the measured ${ }^{2} \mathrm{H}\left({ }^{6} \mathrm{He},{ }^{7} \mathrm{Li}\right) \mathrm{n}$ angular distribution [8]]. The ${ }^{6} \mathrm{He}(p, \gamma){ }^{7} \mathrm{Li}$ cross section was then deduced with the newly extracted ${ }^{7} \mathrm{Li}$ proton spectroscopic factor [Q]. According to charge symmetry of Mirror nuclei, we can deduce the proton spectroscopic factor from the neutron spectroscopic factor or the nuclear asymptotic normalization coefficient (ANC) of its mirror nucleus. The astrophysical ${ }^{6} \mathrm{Li}(p, \gamma){ }^{7} \mathrm{Be} \mathrm{S}$-factors were derived with the deduced ${ }^{7} \mathrm{Li}$ neutron spectroscopic factor from ${ }^{7} \mathrm{Li}\left({ }^{6} \mathrm{Li},{ }^{7} \mathrm{Li}\right){ }^{6} \mathrm{Li}[[]]$. We determined the ${ }^{8} \mathrm{Li}(p, \gamma){ }^{9} \mathrm{Be}$ cross section by the angular distributions of ${ }^{2} \mathrm{H}\left({ }^{8} \mathrm{Li},{ }^{9} \mathrm{Be}\right) n$ and ${ }^{13} \mathrm{C}\left({ }^{9} \mathrm{Be},{ }^{8} \mathrm{Li}\right){ }^{14} \mathrm{~N}$. The spectroscopic factor of ${ }^{9} \mathrm{Be}={ }^{8} \mathrm{Li}+p$ was extracted to be $0.64 \pm 0.21$ with the angular distribution of ${ }^{2} \mathrm{H}\left({ }^{8} \mathrm{Li},{ }^{9} \mathrm{Be}\right) n$ reaction in 2006 [山]]. However, Camargo et al. extracted the proton spectroscopic factor of ${ }^{9} \mathrm{Be}$ to be $1.50 \pm 0.28$ with the measured angular distribution of ${ }^{9} \mathrm{Be}\left({ }^{8} \mathrm{Li},{ }^{9} \mathrm{Be}\right){ }^{8} \mathrm{Li}$ elastic transfer reaction in 2008 [ए]]. Their value was larger than ours by a factor of 2 . To clarify this discrepancy, we measured the angular distribution of ${ }^{13} \mathrm{C}\left({ }^{9} \mathrm{Be}\right.$, $\left.{ }^{8} \mathrm{Li}\right){ }^{14} \mathrm{~N}$ at $\mathrm{HI}-13$ tandem accelerator this year, and the proton spectroscopic factor of ${ }^{9} \mathrm{Be}$ was extracted to be $0.73 \pm 0.15$. The result proved the correctness of the ${ }^{9} \mathrm{Be}$ proton spectroscopic factor and ${ }^{8} \mathrm{Li}(p, \gamma){ }^{9} \mathrm{Be}$ cross section extracted from the ${ }^{2} \mathrm{H}\left({ }^{8} \mathrm{Li},{ }^{9} \mathrm{Be}\right)$ n reaction.

The ${ }^{4} \mathrm{He}(2 n, \gamma)^{6} \mathrm{He}(p, n)^{6} \mathrm{Li}$ reactions were supposed to be a way to increase the primordial ${ }^{6} \mathrm{Li}$ abundance. The angular distributions of ${ }^{6} \mathrm{He}(p, n)^{6} \mathrm{Li}$ reaction leading to the ground and 3.563 $\mathrm{MeV} 0^{+}$states of ${ }^{6} \mathrm{Li}$ have been measured using the ${ }^{6} \mathrm{He}$ radioactive beam at energy of $4.17 \mathrm{AMeV}$ [12]. The experiment reveals the proton-neutron halo structure of the secondary excited state of ${ }^{6} \mathrm{Li}$, which was predicted by Arai et al. [[13]. The dependence of the cross section as a function of energy in the center of mass frame is calculated with the nuclear reaction code Talys [प]]]. In the similar way, the excitation functions of $\left.{ }^{6} \mathrm{He}(d, n){ }^{7} \mathrm{Li},{ }^{8} \mathrm{Li}(p, d)\right)^{7} \mathrm{Li},{ }^{8} \mathrm{Li}(p, t){ }^{6} \mathrm{Li},{ }^{8} \mathrm{Li}(d, p){ }^{9} \mathrm{Li}$, ${ }^{8} \mathrm{Li}(d, n){ }^{9} \mathrm{Be},{ }^{7} \mathrm{Be}\left(d,{ }^{3} \mathrm{He}\right){ }^{6} \mathrm{Li}$ were deduced by the measured cross section in inverse kinematics

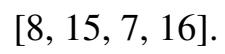

The cross section of ${ }^{9} \mathrm{Be}(p, \alpha)^{6} \mathrm{Li}$ was extracted by means of the Trojan horse method (THM) using the ${ }^{2} \mathrm{H}\left({ }^{9} \mathrm{Be}, \alpha{ }^{6} \mathrm{Li}\right) \mathrm{n}$ reaction at a beam energy of $22.35 \mathrm{MeV}$ [ㅁ] ]. In the analysis, ${ }^{9} \mathrm{Be}+$ ${ }^{2} \mathrm{H} \rightarrow{ }^{9} \mathrm{Be}+p+n$ was assumed as an intermediate process in the quasifree condition, and the deuteron is used as "Trojan Horse" nucleus. The cross section relation between two-body and three-body reactions given by THM was applied to extract the energy dependance of the two body cross section. The $\mathrm{S}(0)$ factor was then determined to be $21.0 \pm 0.8 \mathrm{MeV} \mathrm{b}$, which was in good agreement with the direct experiments [ए], प9]. Furthermore, the electron screening potential energy $U_{e}$ was also been extracted by comparing the direct and THM data [17]]. 


\section{Network calculations}

With the cross sections of the above mentioned reactions, the thermonuclear reaction rates $\langle\sigma v\rangle$ can be calculated by

$$
\langle\sigma v\rangle=\left[\frac{8}{\pi \mu}\right]^{1 / 2}[k T]^{-3 / 2} \int \sigma(E) E e^{-E / k T} d E
$$

where $\mu$ is the reduced mass of the system, $\mathrm{E}$ is the energy in the center of mass system, $k$ is the Boltzmann constant, and $\mathrm{T}$ is the temperature in Kelvin.

The reaction network calculations have been done with a modified code based on the computational routines of Wagoner [四]. The reactions involving four different nuclei may be depicted as

$$
N_{i}\left({ }^{A_{i}} Z_{i}\right)+N_{j}\left({ }^{A_{j}} Z_{j}\right) \leftrightarrows N_{k}\left({ }^{A_{k}} Z_{k}\right)+N_{l}\left({ }^{A_{l}} Z_{l}\right)
$$

$N_{i, j, l, k}$ is the number of nuclear $i, j, l, k$. The rate for the abundance change of any nucleus $i$ is determined by the rate equation

$$
\frac{d Y_{i}}{d t}=\sum_{j, k, l} N_{i}\left(-\frac{Y_{i}^{N_{i}} Y_{j}^{N_{j}}}{N_{i} ! N_{j} !}[i j]_{k}+\frac{Y_{l}^{N_{l}} Y_{k}^{N_{k}}}{N_{l} ! N_{k} !}[l k]_{j}\right),
$$

where $Y_{i}$ is the mass fraction contained in nucleus $i,[i j]_{k}$ represents the reaction rate for the reaction between $i$ and $j$, including the decay rate of nuclide $i$. The sum in Eq. (3.3) includes all reactions involving nucleus $i$.

The evolution of the abundance for ${ }^{6} \mathrm{Li},{ }^{7} \mathrm{Li}$ and ${ }^{7} \mathrm{Be}$ of our calculations is shown in Figure ㅁ. In general, it was found that the added reactions slightly changed the lithium abundance at $\mathrm{t}<$ $1000 \mathrm{~s}$, but have minimal effect at $\mathrm{t}>1000 \mathrm{~s}$. This may be attributed to the small binding energies of lithium. The detailed study showed that ${ }^{6} \mathrm{Li}(p, \alpha)^{3} \mathrm{He}$ could have large effect for destroying ${ }^{6} \mathrm{Li}$,

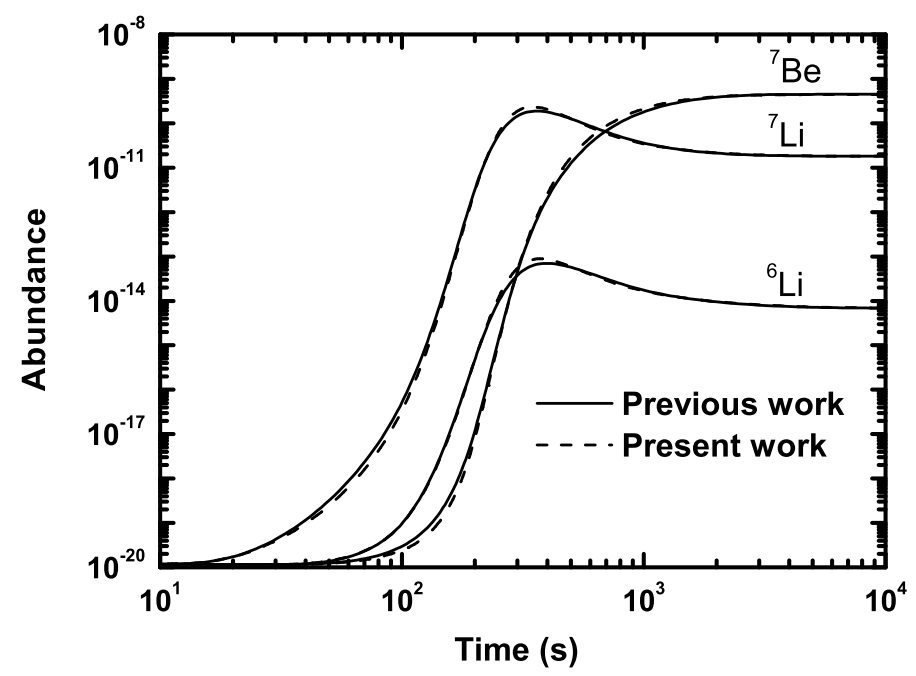

Figure 2: Nuclear abundance as a function of BBN times. 
leading to the disappearance of the effect of the added reaction. The abundance of ${ }^{7} \mathrm{Li}$ is sensitive to the ${ }^{2} \mathrm{H}(p, \gamma)^{3} \mathrm{He},{ }^{3} \mathrm{He}(\alpha, \gamma){ }^{7} \mathrm{Be}$, and ${ }^{7} \mathrm{Li}(p, \alpha){ }^{4} \mathrm{He}$ reaction rates. Unfortunately, the existing experimental data have large uncertainties.

\section{Conclusion and discussion}

We have determined the rates of fourteen reactions involving lithium isotopes in the past ten years, and the BBN calculation with the expanded network shows that the attempts to solve the lithium abundance problem by measuring all the lithium involving reactions are failed. A series of failures indicate that the lithium puzzle may not be arising due to insufficient knowledge of nuclear reaction, although some reactions, such as the reactions ${ }^{2} \mathrm{H}(p, \gamma){ }^{3} \mathrm{He},{ }^{3} \mathrm{He}(a, \gamma){ }^{7} \mathrm{Be}$ and ${ }^{7} \mathrm{Li}(p, \alpha){ }^{4} \mathrm{He}$ mentioned above, need be determined more accurately.

It is very important to investigate the BBN model more carefully. The improvements of the lithium observations in very metal poor stars could also help us to solve the lithium problem. Very recently, J. C. Howk et al. reported their observation of interstellar lithium in the low-metallicity small Magellanic cloud [20]. The present-day ${ }^{7} \mathrm{Li}$ abundance is nearly equal to the BBN predictions. Measurements of ${ }^{6} \mathrm{Li}$ in stellar atmospheres are extremely difficult because the stellar line broadening is well in excess of the isotope shift. Their works give us a clue to understand the lithium puzzles.

\section{References}

[1] M. Spite, F. Spite, Nature, 297 (1982) 483-485.

[2] A. Coc, E. Vangioni-Flam, P. Descouvemont et al. Astrophys J 600 (2004) 544-552.

[3] M. Asplund, D. L. Lambert, P. E. Nissen et al. Astrophy J 644 (2006) 229-259.

[4] R. Wagoner, Astrophys J 18 (1969) 247-295.

[5] J. Su, Z. H. Li, B. Guo et al. Chin Phys Lett 27 (2010) 052101.

[6] Z. H. Li, B. Guo, Y. J. Li et al. Plasma Science and Technology 14 (2012) 488-491.

[7] Z. H. Li, W. P. Liu, X. x. Bai et al. Phys Rev C 71 (2005) 052801(R).

[8] Z. H. Li, E. T. Li, B. Guo et al. Eur Phys J 44 (2010) 1-5.

[9] E. T. Li, Z. H. Li, J. Su et al. Chin Phys Lett 28 (2011) 052102.

[10] J. Su, Z. H. Li, B. Guo et al. Chin Phys Lett 23 (2006) 55-57.

[11] O. Camargo, V. Guimarães, R. Lichtenthäler et al. Phys Rev C 78 (2008) 034605.

[12] Z. H. Li, W. P. Liu, X. X. Bai et al. Phys Lett B 527 (2002) 50-54.

[13] K. Arai, Y. Suzuki, K. Varga, Phys Rev C 51 (1995) 2488-2493.

[14] A. J. Koning, S. Hilaire, S. Goriely, Nucl Phys A 810 (2008) 13-76.

[15] Y. J. Li, Z. H. Li, B. Guo et al. Chin Phys Lett 25 (2008) 455-457.

[16] S. Zeng, W. P. Liu, Z. H. Li et al. Chin Phys Lett 22 (2005) 2219-2221.

[17] Q. G. Wen, C. B. Li, S. H. Zhou et al. Phys Rev C 78 (2008) 035805. 
[18] A. J. Sierk and T. A. Tombrello, Nucl Phys A 210 (1973) 341-354.

[19] D. Zahnow, C. Rolfs, S. Schmidt et al. Z Phys A 359 (1997) 211-218.

[20] J. C. Howk, N. Lehner, B. D, Fields and G. J. Mathews, Nature 489 (2012) 121-123. 